\title{
Providing Public Access to Native Title Records: Balancing the Risks Against the Benefits
}

\author{
Pamela McGrath
}

\section{Introduction}

Since the establishment of the Native Title Act 1993 (Cth) ("Native Title $\left.A c t^{\prime}\right)$, Aboriginal and Torres Strait Islander peoples have lodged close to 1,800 native title claimant applications; 360 of these have since been determined, 40 by trial. Many claims were ultimately withdrawn; however, 266 remain on foot. ${ }^{1}$ A further 41 compensation applications have been filed. As a result of these many legal proceedings, the Federal Court of Australia and the National Native Title Tribunal (NNTT) are now in possession of an enormous number of records that contain information about many thousands of Aboriginal and Torres Strait Islander persons, both living and deceased. ${ }^{2}$

\footnotetext{
1 As at 5 June 2017, 1,806 claimant applications had been filed since 1993. National Native Title Tribunal (NNTT), Search Native Title Applications, Registration Decisions and Determinations (5 June 2017) National Native Title Tribunal <http:/www.nntt.gov.au/searchRegApps/NativeTitleClaims/ Pages/default.aspx>.

2 The NNTT and Federal Court are not alone in possessing such information; over the years, state governments and respondent parties have accumulated similar collections of native title records. The collections of state governments are likely far larger, as during mediations towards consent determinations, they are provided with copies of evidence such as connection reports and genealogies that are ultimately never submitted to the Federal Court or NNTT.
} 
Native title records are exceptional in both scale and scope and are as unique as the property rights they evidence. As legal records, they are an account of the administration of justice, but they also have broader historical and cultural importance. Collectively, they tell the story of the implementation of one the most significant political interventions in colonial relations since 1788, when Arthur Phillip planted a British flag on the land of the Eora Nation at the place now known as Sydney Cove. Perhaps more importantly, they contain extensive documentation of Aboriginal and Torres Strait Islander peoples' families, histories and cultural practices in relation to land, tendered as proof of asserted rights and interests, and constitute a unique body of research that is not available elsewhere.

Indeed, the onerous evidentiary requirements of the Native Title Act have resulted in, albeit unintentionally, one of the most substantial governmentsponsored research efforts ever undertaken with Indigenous Australians. At the outset, applicants are required to submit genealogical, cultural and historical information that demonstrates their traditional connections to land; ${ }^{3}$ as proceedings progress to mediation or litigation, more detailed evidence is required to persuade both the Federal Court and respondent parties of the veracity of their claims.

How should native title records be managed into the future? Who should be able to access them, and for what purposes? Developing appropriate policies for the management and archiving of native title records is a necessary but onerous responsibility for the Federal Court and NNTT. Part of the challenge lies in the fact that such protocols must address two key imperatives that are not always compatible: the need to ensure public

3 Evidence is required to demonstrate that native title exists, as defined by s 223 of the Native Title Act, which states that:

The expression native title or native title rights and interests means the communal, group or individual rights and interests of Aboriginal peoples or Torres Strait Islanders in relation to land or waters, where:

(a) the rights and interests are possessed under the traditional laws acknowledged, and the traditional customs observed, by the Aboriginal peoples or Torres Strait Islanders; and

(b) the Aboriginal peoples or Torres Strait Islanders, by those laws and customs, have a connection with the land or waters; and

(c) the rights and interests are recognised by the common law of Australia. 
confidence in the administration of justice and the need to respect the concerns of Aboriginal and Torres Strait Islander peoples in relation to personal or culturally sensitive information. ${ }^{4}$

Using the example of a series of research reports produced by the NNTT between 1995 and 2012, this chapter explores some of the intersecting legal and ethical interests that complicate the possibility of making native title records publicly available through either archives or publication. Rather than applying a strictly legal reading of what is or is not permissible, I advocate for a risk-based approach that prioritises the impacts of information management policies on Indigenous Australians and their relationships with others. Identification of attendant risks requires, I argue, meaningful consultation with relevant Aboriginal and Torres Strait Islander peoples and their informed consent for any mitigation strategies.

But is this practically achievable given the potential cost of undertaking consultation and the limited and ever-diminishing resources available to the Federal Court and the NNTT? An alternative approach proposed here is to ensure that native title records held by the Federal Court, the NNTT and the National Archives of Australia (NAA) are at the very least readily discoverable. Facilitating public discoverability as the first step in developing comprehensive management protocols will reduce the legal and cultural risks associated with unfettered public access while ensuring that native title records can always be found if and when they are wanted.

\section{Weighing Up the 'Public' Interest}

Any decision to make native title records publicly available requires consideration of a broader public interest. At first glance, the cultural, educational and historical significance of native title records implies a public interest that should be supported with public access. Yet the highly personal nature of much of the information these records contain, and the circumstances under which they were obtained, raise some very good reasons as to why their availability should, in fact, be limited.

4 Ian Irving, 'The Challenges of Managing Documents Related to Native Title Hearings' in Pamela Faye McGrath, Ludger Dinkler and Alexandra Andriolo (eds), Managing Information in Native Title (MINT) (Australian Institute of Aboriginal and Torres Strait Islander Studies, 2015) 53. 
The 'public interest' is not a clearly defined legal concept, but, rather, is treated by the law as 'flexible enough to respond to the facts and circumstances of any particular case'. ${ }^{5}$ When used in a statute, as it is in the Native Title Act, the term 'public interest' derives its content from 'the subject matter and the scope and purpose' of the legislation in which it appears. ${ }^{6}$ Among the possible public interest matters that may be relevant in the context of native title, ensuring the proper administration of government and the promotion of open justice are among the most compelling. ${ }^{7}$ However, the public interest must also be considered in relation to the subject, scope and purpose of the Privacy Act 1988 (Cth) ('Privacy $A c t$ ').

Further, there is a need for both privacy and the public interest to be considered against the cultural and customary concerns of Aboriginal and Torres Strait Islander peoples. Ultimately, it falls on the Native Title Registrar to decide on this balance.

Under the Native Title Act, the Native Title Registrar may keep other records and information as he or she considers appropriate, and make those records available to the public. ${ }^{8}$ The Native Title Registrar must not make information available to the public if he/she considers that it would not be in the public interest to do so. And when considering the public interest, the Native Title Registrar must take into account the cultural and customary concerns' of Aboriginal peoples and Torres Strait Islander peoples. ' The term 'cultural and customary concerns' is undefined in the Native Title Act and can reasonably be interpreted as potentially including concerns about the management of personal or culturally sensitive information.

This raises a critical question about the extent to which the interests of Aboriginal and Torres Strait Islander persons and peoples are usually accommodated within consideration of a broader 'public' interest. Aboriginal and Torres Strait Islander persons are undoubtedly members

5 Law Institute of Victoria, Submission 22, cited in Australian Law Reform Commission, Serious Invasions of Privacy in the Digital Era, Discussion Paper 80 (2014) $116<$ https://www.alrc.gov.au/ publications/serious-invasions-privacy-dp-80>.

6 Hogan v Hinch (2011) 243 CLR 506, [31], cited in Australian Law Reform Commission, above n 5 .

7 For a list of common values, freedoms and matters of public interest, see Australian Law Reform Commission, above $\mathrm{n} 5,30$.

8 Native Title Act ss 98A (1) and (2).

$9 \quad$ Native Title Act s 98A. 
of the public, but as a social category, they are a 'persistent minority'. ${ }^{10}$ Persistent minorities rarely have their way in processes of democratic decision-making, which has profound consequences for their ability to 'make the larger world in which they live a home for themselves'. ${ }^{11}$ In the words of Noel Pearson, it is the structural problem of the 3 per cent 'mouse' of Indigenous Australia contending with the 97 per cent 'elephant' of the rest of the population: 'The 3 percent can never really get the 97 percent elephant to behave in a way that treats the mouse with dignity as citizens'. ${ }^{12}$

It appears that the Native Title Act is in fact structured towards addressing this demographic disadvantage. The obligation for the Registrar to consider the cultural and customary concerns of Aboriginal and Torres Strait Islander people as a matter of public interest is clearly aimed at supporting Indigenous Australians to have a greater say over the fate of information about themselves, their families and their land. It follows that the assumption at the heart of this intention-namely that Aboriginal and Torres Strait Islander peoples are a segment of the Australian public whose interests, in this context, are to be given priority—should be a central consideration in the development of protocols for the management of native title records into the future.

But what does this look like in practice? Drawing on the lessons learned from developing management protocols for the NNTT research reports, I am advocating for a process that puts the opinions and perspectives of Aboriginal and Torres Strait Islander peoples at the centre of any analysis of the risks and benefits associated with creating public access to native title records. The discussion that follows does not reflect current NNTT or court policy in relation to managing and creating access to native title records. ${ }^{13}$ Rather, it offers a particular perspective on the issues that is grounded in my many years of research and policy experience in this area.

10 Thomas Christiano, 'Democratic Equality and the Problem of Persistent Minorities' (1994) 23(3) Philosophical Papers 169.

11 Jeff Spinner-Halev, Enduring Injustice (Cambridge University Press, 2012) 133.

12 Noel Pearson, 'Keynote Address: An Agenda for Indigenous Empowerment' (Speech delivered at the Royal Australian and New Zealand College of Psychiatrists Annual Congress, Brisbane, 6 May 2015) 9 <http://capeyorkpartnership.org.au/news/an-agenda-for-indigenous-empowerment/>.

13 A small number of the research reports are subject to directions from a Tribunal Member restricting their disclosure made under s 94L or s 136GD of the Native Title Act. This special category of report requires a unique management regime and is not dealt with in any detail here. 


\section{The NNTT Research Reports}

Among the many thousands of native title records held by the NNTT is a small but significant collection of 280 research reports that were produced by NNTT researchers (employees of the Australian Public Service) during the period between 1995 and 2012. These research reports were generally commissioned for one of two statutory purposes: to assist parties in relation to native title proceedings under $s 78$ of the Native Title Act; and to assist the NNTT with the mediation of applications under s 108(2) of the Native Title Act.

The research reports are substantial documents that bring together, often for the first time, copies of publicly available historical and ethnographic ('ethno-historical') sources about particular Aboriginal or Torres Strait Islander groups in relation to particular areas. The ethno-historical items they include were authored by many different kinds of people-explorers, anthropologists, linguists, pastoralists and the like-the vast majority of whom were non-Indigenous. Typically, the sources range from extracts of early maps of 'tribal' or 'language' boundaries, journals and field notes, mission and government records, books, newspaper articles and academic papers to published oral histories of Aboriginal and Torres Strait Islander individuals and families.

For each research report, the ethno-historical materials are accompanied by a summary chapter written by NNTT researchers. These provide background information about the authors of the ethno-historical materials and the circumstances in which they collected their data. Some of the summary chapters include focused discussion about specific matters of contention-for example, the location of a boundary between two groups or the identity of a local ancestor. ${ }^{14}$

The amount of information contained in the research reports is substantial. To give a typical example, a research report prepared in 2001 relates to three different native title groups and includes items spanning a time period of 102 years. Specifically, it contains:

14 Each summary chapter also typically includes a list of sources viewed but not cited and a bibliography. Some of these bibliographies have already been published by the NNTT as standalone documents and are currently available on the NNTT website (although the summary chapters themselves are not); see National Native Title Tribunal, Publications (2016) National Native Title Tribunal <http://www.nntt.gov.au/News-and-Publications/Pages/Forms-and-Publications.aspx>. 
- a summary chapter of 52 pages

- 32 ethno-historical map appendices

- three maps showing relevant native title claims

- 33 ethno-historical document appendices, ranging between one and 30 pages in length.

This particular report also names 90 different Aboriginal persons, some of whom are still living, and includes numerous statements about the location of traditional territorial boundaries, sites of ritual significance and commentaries on the practice of culture and custom (including references to gender-restricted ceremonies). The unpublished ethnohistorical sources included in the report came from five different archives located in four different Australian cities and previously published ones were obtained from a number of different libraries and secondhand booksellers.

With the hard work of locating, scanning and aggregating all this material already done, each research report provides immediate access to a research literature that, even though already publicly available, was previously out of reach to anyone without the necessary time, money and expertise to search for it themselves. In this sense, the potential value of the research reports to Aboriginal and Torres Strait Islander people and their researchers, lawyers and advocates, as well as respondents to their claims, cannot be understated. They are also of potential value to academics and members of the public who, for other reasons, are interested in understanding the indigenous social and cultural landscapes of particular areas.

That said, the research reports are not without their flaws. The ethnohistorical materials they contain are not comprehensive and vary considerably in scope and quality. There are some national sources, such as Norman Tindale's Aboriginal Tribes of Australia and David Horton's Encyclopaedia of Aboriginal Australia, that are prominently featured in almost every research report, while other significant but less well-known and accessible materials are overlooked. ${ }^{15}$

15 A number of research reports include transparency maps showing the location of the underlying native title claim in relation to both Tindale and Horton's accounts of group boundaries; Norman Tindale, Aboriginal Tribes of Australia: Their Terrain, Environmental Controls, Distribution, Limits and Proper Names (University of California Press, 1974); David Horton (ed), Encyclopaedia of Aboriginal Australia (Aboriginal Studies Press, 1994). 
Further, the analysis contained in the summary chapters does not take into account the perspectives of contemporary native title claimants. This was a deliberate decision on the part of the NNTT, aimed at ensuring that the objective neutrality of the NNTT was preserved and that the reports did not stray into the territory of providing expert opinion. As a consequence, the sources are presented without critical assessment of their reliability or the weight they should be given when considering any underlying native title rights.

The NNTT was alert to these shortcomings at the time they were drafted and acknowledged them with disclaimers that nicely highlight some of the attendant risks and benefits associated with their possible publication. Among other things, the disclaimers note that the research reports were prepared within short time frames, are not comprehensive, and that the inclusion of particular ethno-historical items does not constitute an endorsement of those items by the NNTT. Where the research reports contain names of deceased Aboriginal people or culturally sensitive information, warning statements are also included.

Despite these limitations, the research reports were, and remain, a valuable if sometimes problematic resource for claimants and respondent parties alike. The preparatory research undertaken for native title claims during the early years of the Native Title Act was extraordinary and involved intensive documentary discovery. Scores of researchers and traditional owners were simultaneously tasked with the job of locating and reviewing thousands of potentially relevant records. The demand on libraries, archives and museums was so intense that the Commonwealth Government funded the Australian Institute of Aboriginal and Torres Strait Islander Studies (AIATSIS) to establish a Native Title Access Unit to deal with the surge in requests from native title groups and respondent parties for searches of their collection. ${ }^{16}$

The NNTT research reports were of great assistance in this effort, saving everyone involved considerable time and money. Despite their variable reliability, they were useful for examining the factual basis of applications, preparing documentation for registration testing and negotiating boundaries between overlapping claims. A crucial starting point for native title applicants, they also provided reassurance to non-Indigenous

16 Copies of many of the ethno-historical materials included in the NNTT research reports were provided by the Australian Institute of Aboriginal and Torres Strait Islander Studies (AIATSIS). 
respondent parties sceptical of the very fact of prior occupation ('yes, there is evidence that Aboriginal people actually lived here'). In light of this utility, the research reports were usually distributed widely to everyone involved in a matter-anthropologists, claim lawyers, respondents and state government officials alike.

They were especially valuable to researchers located in remote areas during the early years of native title when digital technologies had yet to become commonplace. When I worked as a researcher in the Tom Price office of the Pilbara Native Title Service in the early 2000s, Google was barely two years old, broadband internet was in its infancy and our unreliable dial-up connection was torturously slow. All the archives I needed to access for the claims I was researching were located many thousands of kilometres away in Perth, Adelaide and Canberra, and the native title representative body I worked for could only afford to employ a single historian, who undertook background research for more than 30 different claims. In these circumstances, the NNTT research reports were a godsend, providing my colleagues and me with access to crucial pieces of evidence that would otherwise have been beyond our reach.

However, these reports undoubtedly mean the most to those Aboriginal and Torres Strait Islander peoples whose histories are contained in their pages. For many groups, the research reports were the first time that a survey of ethno-historical sources about their people had been undertaken, and/or the first time they had access to these sources themselves. Far from being neutral documents, the collective knowledge they contain about country, culture, kin and the impact of colonial settlement affords them a degree of emotional and political power that resonates well beyond their original purpose. Their contents have the potential to confirm or deeply disturb an individual's fundamental sense of self and where they belong in the world, generating joy, grief, shame, anger and argument in turn and altering both an individual and shared sense of social reality. Disclaimers mean little in such circumstances. Rather, sensitive and respectful attention is required in the development of any plans for the future management of these valuable assets. 


\section{Managing Valuable Native Title Records}

It is now 25 years since the first NNTT research report was produced, and there is a pressing need to have them archived. This requires consideration of, among other things, the level of public access allowed once they are lodged with the NAA. For many reasons, this will not be a straightforward undertaking.

The research reports remain sought-after assets, even though the NNTT has not produced a research report since 2012 and many of the older research reports are now effectively redundant because native title has been settled in the areas they discuss. They continue to be used by parties involved in native title proceedings, and every year the NNTT receives a number of requests for copies of existing reports from members of native title groups, their legal representatives, respondent parties and the Federal Court. Requests also occasionally arrive from other interested Aboriginal and Torres Strait Islander persons and members of the public. Once archived, such requests will also be received by NAA.

There are some significant differences in the obligations of the NNTT and the Federal Court in relation to archiving of legal records, despite an overlap in the records they hold (both hold some of the same kind of records, as well as records relating to the same proceedings). ${ }^{17}$ The NNTT and the Federal Court operate under different NAA record authorities and are dealt with differently under the Privacy Act. Consequently, each organisation must develop its own approach to archiving and accessing native title records, and responsibility for developing management policies for the NNTT's research reports lies with NNTT alone. ${ }^{18}$

The NNTT's policy for managing access to the research reports has been revised a number of times over the course of the last two decades. At times, the research reports have been made widely available, including on the NNTT website, with little control over their distribution. ${ }^{19}$ At other

17 Prior to legislative amendments to the Native Title Act in 2012, the NNTT was responsible for case management of native title claims and, therefore, both organisations are in possession of case management files. Similarly, as it currently stands, the NNTT undertakes registration testing for native title applications for which the Federal Court provides case management, leading to a situation where both organisations are in possession of records relevant to a particular matter.

18 Federal Court of Australia, Annual Report 2014-15 (Federal Court of Australia, 2016) 21, 60 $<$ http://www.fedcourt.gov.au/digital-law-library/annual-reports $>$.

19 Copies of some of the research reports are held in the AIATSIS collection. 
times, a very cautious approach has been taken, and their distribution has been carefully controlled and monitored. The current NNTT policy deals with requests for access on a case-by-case basis, but, in principle, aims to provide native title groups and their representatives with all relevant information held by the NNTT. Facilitating access requests from members of the public is complicated by the absence of a direct interest.

Developing appropriate access protocols is an issue that NNTT continues to wrestle with. When I was appointed NNTT Research Director in 2015, I was given responsibility for reviewing the access policy for the research reports and developing a plan for their longer term management. Pre-armed with firsthand experience of their great utility and value as tools for native title researchers, I embraced the task with enthusiasm and immediately started exploring the possibility of making them more readily available, perhaps through open-access online publication. It seemed to me that such useful documents should be made more widely available and that this could be done in a way that encouraged self-service and reduced the administrative burden of the NNTT.

After 12 months of scoping the possibilities, delving into countless layers of legal, cultural and ethical complexity, I was prepared to admit that my initial enthusiasm for an 'open access' approach to publishing the NNTT research reports may have been pre-emptive. I had come to appreciate that, even though copies of most of the research reports are already in circulation and contain information that is already publicly available, increasing public access to these unique knowledge assets is not without risk. These risks are for the most part borne by Aboriginal and Torres Strait Islander people alone. Moreover, it has become increasingly clear that the attendant risks will not be effectively mitigated or resolved by simply applying a strict interpretation of what is legally permissible. A different approach is required that more fully considers the possible social impacts of the research reports within their home communities. 


\section{Assessing the Risks and Benefits of Creating Public Access}

While most of the information contained in the research reports is freely available elsewhere, this fact alone does not diminish the potential risks associated with allowing public access to them, and the task of developing appropriate policies for their ongoing management has proven to be complex.

There is a degree of ambiguity in the Native Title Act that may be interpreted as providing for, or at least not preventing, public access to the research reports. As previously noted, the Registrar alone is empowered to make decisions about the provision of native title information to the public, and, when doing so, must take into consideration matters of public interest.

Given the historical and educational value of the research reports, there would seem to be a clear public benefit to making them available. However, the Native Title Act does not confer any specific educational purpose on the NNTT or Native Title Registrar, and the original purpose for which research reports were prepared was not educational. Nevertheless, the Native Title Act does not necessarily preclude publication of the research reports for educative or research purposes, and ss 78(1)b and 78 (2)(a) provide for the Native Title Registrar to provide assistance, including research assistance, to any person at any stage of a proceeding in matters relating to that proceeding.

But do the potential benefits of providing public access to these records for educational reasons justify the potential risks such access poses to Indigenous Australians? One way of approaching this question, I suggest, is to engage in a process of risk analysis aimed at identifying how the publication of native title records will impact on Aboriginal and Torres Strait Islander peoples and persons, their communities and their relationships with other Australians. The explicit intention of such an analysis should be to inform the development of policies that not only minimise risk but also empower and strengthen key relationships to fully realise the conciliatory intent of the Native Title Act. ${ }^{20}$

20 The preamble of the Native Title Act makes it clear that the intention of the legislation is to 'rectify the consequences of past injustices' and 'ensure that Aboriginal peoples and Torres Strait Islanders receive the full recognition and status within the Australian nation to which history, their prior rights and interests, and their rich and diverse culture, fully entitle them to aspire' and to 'further advance the process of reconciliation among all Australians'. 
Risk management in government is a highly specialised area that is guided by its own set of rules and requires expertise in its own right. ${ }^{21}$ The approach that I present here does not represent the current policy and practice of the NNTT or the Federal Court, nor is it a definitive assessment of all the attendant risks, their likelihood and/or their potential consequences. Rather, it sets out some basic principles of risk assessment that might be usefully brought to bear on the development of policies for the management of native title records.

A common approach to assessing risk involves applying a framework that identifies the possible consequences of a particular course of action, articulates the likelihood and severity level of risk for particular stakeholders, and develops strategies to mitigate those risks. Risk oversight and management are a key part of the corporate governance of both the Federal Court and NNTT. Both institutions are committed to an organisational culture that supports the identification, analysis, assessment, treatment, monitoring and review of all strategic, professional, reputational, personnel, political and operational risks. These include not only risks to the institutions themselves, but also to their stakeholders. ${ }^{22}$

Needless to say, when it comes to native title matters, it is Aboriginal and Torres Strait Islander peoples who are the primary stakeholders, and the strategic, reputational and political risks they face should be paramount in any consideration of the impacts of creating public access to native title records. This requires consideration not only of impacts internal to Indigenous families and communities, but also risks posed to Indigenous people's relationships with others. In the context of native title, those relationships are between Aboriginal and Torres Strait Islander individuals (as represented by their prescribed bodies corporate) and the rest of the world, with the NNTT and the Federal Court (as representatives of government) playing a central role in the mediation of that relationship.

To put it another way, the failure of relationships between native title holders and the NNTT or the Federal Court will have ramifications for the broader relationship between Indigenous Australians and government, and, as such, any actions that potentially undermine this relationship warrant thoughtful consideration.

21 The Federal Court and NNTT are guided in their approach to risk management by the Australian/ New Zealand Risk Management Standard (AS/NZS ISO 31000:2009) and the Commonwealth Risk Management Policy 2014. Federal Court of Australia, 2016-2020 Corporate Plan (Federal Court of Australia, 2016) 39 <http://www.fedcourt.gov.au/about/corporate-information/corporate-plan>.

22 Ibid. 
Even though they are just a fragment of the entire collection of native title records held by the Federal Court and the NNTT, assessing the risks associated with creating public access to the NNTT research reports is no small task. The risk profile of each research report will vary because each is unique in purpose, scope and methodology and deals with a distinct group or groups of Aboriginal and Torres Strait Islander peoples with their own unique laws and customs. And although much of the information they contain is already available elsewhere, and many of the native title proceedings they relate to have been finalised, the multidimensional nature of the information they contain means that there are potentially many legal and cultural issues to be considered. For the purposes of this paper, I focus on four: copyright, personal information, culturally sensitive information and out-of-date or inaccurate information.

\section{Copyright}

Copyright is perhaps one of the more straightforward issues to be considered in relation to making native title records publicly available. Nevertheless, for reasons explained below, copyright is a potential impediment to publication (ie, reproduction as opposed to access) and has a significant impact on the ability of the NNTT to reproduce the research reports in their entirety in a public forum.

As previously noted, the summary chapter of each research report is an original piece of work that is copyrighted to either the NNTT or the Commonwealth of Australia, and which the NNTT, therefore, has the right to publish. However, each of the appendices has its own unique provenance and copyright remains with the original copyright holder (except where they are out of copyright). The NNTT has never sought permission to use these materials for any purpose other than for their original purpose-namely, to assist with native title claim applications or mediations - and therefore, cannot republish them without first obtaining the permission of the copyright holder. ${ }^{23}$

23 The NNTT operates under the Federal Court's GovCopy licence. The GovCopy licence is for organisations that are not part of, or have not been authorised by, the Crown but are characterised as administrative bodies that are funded by taxpayers. Organisations with a GovCopy licence may use up to 10 per cent of copyright material. This includes reproductions and communications, including internal emailing and uploading of copyright material to intranets. For more information, see: Copyright Agency, Your Organisation and Copyright (2015) CopyrightAgency <http://copyright. com.au/wp-content/uploads/2015/05/CopyrightAgency_GovCopy_FactSheet.pdf>. 
With the total number of appendices in the thousands, it is simply not feasible for the NNTT to seek permission to republish ethno-historical sources from all copyright holders. However, this does not preclude the possibility of making them publicly available through an archive, or publishing the summary chapters as standalone documents (as the copyright for these is held by the NNTT). Although the utility of the summary chapters would be somewhat constrained if they were published without copies of the ethno-historical items to which they refer, for some language groups they remain the only summaries of ethno-historical materials ever prepared and are potentially of value in their own right.

\section{Personal and Sensitive Information}

One of the more complex issues that arises when considering the consequences of creating public access to the research reports, and native title records generally, relates to the management of personal privacy. 'Personal information' as it is defined in the Privacy Act refers to information or opinion about an individual that enables that individual to be identified. 'Sensitive information' is a category of personal information that includes information or opinion about, among other things, an individual's racial or ethnic origin, political opinions and religious and philosophical beliefs. ${ }^{24}$ The Australian Privacy Principles apply to this kind of information, regardless of whether the individual concerned is alive or deceased.

The research reports are replete with information that is of a personal nature. In some cases, they contain photographs of named individuals, living and deceased, and opinion about their racial origins. Others include lists of individuals named in ethno-historical sources, some of whom are still living, alongside facts about language and tribal affiliation. Needless to say, such information is related to the race, ethnicity, political attitudes, and religious and philosophical beliefs of those identified and falls under the category of sensitive information.

24 Office of the Australian Information Commissioner, Australian Privacy Principles (January 2014) Office of the Australian Information Commissioner <https://www.oaic.gov.au/privacy-law/privacyact/australian-privacy-principles $>$. 
As is the case with copyright, there are differences in the privacy obligations of the Federal Court and the NNTT in relation to the records they hold. The Privacy Act does not apply to documents and other materials relating to court proceedings; these are dealt with under the Federal Court of Australia Act 1976 (Cth) and the rules made by Judges of the Federal Court. However, unlike the Federal Court, the NNTT and the Native Title Registrar are bound by the Privacy Act and the Australian Privacy Principles in relation to the collection, use, disclosure, management and access of personal information. ${ }^{25}$

The court has considered privacy issues specifically in relation to the NNTT research reports. They determined that, while it is clear that the NNTT may provide research reports to persons outside of the NNTT to assist with a native title application compatible with the Privacy Act, making research reports available to the general public involves a secondary purpose that may invoke the Privacy Principles. In light of this, the Federal Court's current Privacy Policy states that:

The disclosure of [the NNTT research reports], in whole or in part, may be subject to the Privacy Act and/or specific directions made by the NNTT under section 94L or the former section 136F of the Native Title Act. ${ }^{26}$ (emphasis added)

Given this legal uncertainty, one of the options open to the NNTT is to seek a public interest determination from the Information Commissioner in relation to the publication of the research reports. ${ }^{27}$ This would provide clarity on the matter and, if publication were deemed to be compatible with the Australian Privacy Principles, would enable the NNTT to proceed with publication of the research reports indemnified against the risk of legal action over the publication of personally sensitive information.

25 Federal Court of Australia, Privacy Policy (31 March 2014) Federal Court of Australia <http:// www.fedcourt.gov.au/>.

26 Ibid.

27 'A public interest determination (PID) may provide that an act or practice of an APP entity that could otherwise breach an Australian Privacy Principle (APP), or a registered APP code that binds that entity, shall not be regarded as having done so (s 72(2)). An APP entity undertaking that act or practice will not be taken to have contravened s 15 of the Privacy Act (or s 26A if an APP code)': Office of the Australian Information Commissioner, Privacy Public Interest Determination Guide (June 2014) Office of the Australian Information Commissioner <https://www.oaic.gov.au/ agencies-and-organisations/guides/privacy-pid-guide>. 
However, such indemnity does not mitigate the risks for those Aboriginal and Torres Strait Islander persons whose personal information is published. It is precisely in such a situation-where the legality of a particular course of action is unclear or ambiguous and does not adequately accommodate the risks of key stakeholders - that it is useful to shift the focus onto the relationships underpinning the proposed transaction, and the potential risks that any particular course of action poses to those relationships. The failure of the NNTT and the Federal Court to appropriately acknowledge and mitigate such risks may in fact undermine the level of trust between Aboriginal and Torres Strait Islander peoples and government (as represented in this instance by the NNTT and the Federal Court), and is a political risk in its own right.

Despite the fact that most ethno-historical sources used in the research reports are already publicly available, the potential for the personal information they contain to damage an individual's or group's reputations and relationships is very real. This risk is intensified by the fact that the research reports increase the ease with which such information can be discovered. Consider, for example, the potential impact of a single snippet from an ethno-historical source that identifies a particular ancestor as belonging to language group $\mathrm{X}$, despite the fact that their descendants actively identify that person as belonging to language group Y. It won't matter whether the reliability of that particular 'fact' has been debated in court and found wanting; out of context and in the absence of detailed analysis of its veracity, that 'fact' has the potential to damage the status and standing of an entire family, both within their community and more broadly within the Australian society.

Not all native title records containing information of a personal and sensitive nature will carry such risks, and it may be the case that in some instances creating public access to the research reports would be welcome and celebrated by the Indigenous persons and native title groups concerned. But while there are certain kinds of information that are more likely to be of concern than others, there are no fixed criteria by which the likelihood of risk or consent can be predicted by a third party. The only way to identify the likelihood and severity of the substantive risks associated with exposing sensitive personal information for any particular matter is to consult the individuals directly involved; given the amount of personal and sensitive information contained in the research reports, this will be a time-consuming and expensive undertaking. 


\section{Culturally Sensitive Information}

As with information of a personally sensitive nature, the task of identifying and mitigating risks associated with exposure of culturally sensitive information is a complex proposition. Bureaucrats employed by the Federal Court and the NNTT are generally not well placed to understand and assess the attendant risks and, as with personally sensitive information, consultation with relevant Indigenous parties will be required.

The types of culturally sensitive information that are commonly problematic in the public domain are relatively well-known and include, for example, the names or images of deceased individuals; references to male initiation practices; out-of-date terminology; and information that identifies the location of sacred sites. However, the great diversity of cultural practices and historical experiences among the many hundreds of Aboriginal and Torres Strait Islander groups around Australia makes it is impossible to predict the potential for any particular details to breach local cultural protocols.

The hurt individuals and families may experience as a result of the publication of culturally sensitive information is very real. Individuals' relationships with cultural information are profound, as are the possible consequences of failing to control its' distribution appropriately. Breaches of traditional law and custom in relation to cultural information may result in pain, anxiety, illness and, potentially, death, and the people deemed responsible for a breach may be punished by their community. The loss of information and authority in relation to both culture and country, in turn, undermines an individual's cultural status and impedes their ability to reproduce their traditions and, therefore, themselves in very fundamental ways. ${ }^{28}$

The significance of the impacts of exposure and loss of cultural information is underscored by a recent Federal Court decision in the Timber Creek native title compensation case. In 2016, the Ngaliwurru and Nungali peoples were awarded a solatium of $\$ 1.3$ million for hurt arising from damage caused over the loss of their native title rights in an area of approximately 79 hectares in and around the town of Timber

28 Benedict Scambary and Gareth Lewis, 'Sacred bodies and ore bodies: Conflicting commodification of landscape by Indigenous peoples and miners in Australia's Northern Territory' in Pamela F McGrath (ed), The Right to Protect Sites: Indigenous Heritage Management in the Era of Native Title (AIATSIS Research Publications, 2016) 224. 
Creek. Crucial in consideration of the solatium were multiple dimensions of social effect-namely, 'loss of amenities', 'pain and suffering' and 'reputational damage'. Central to the judge's decision was the hurt caused by a loss of cultural reputation among members of neighbouring groups and 'the sense of failed responsibility for the obligation ... to have cared for ... that land' ${ }^{29}$

The consequences of exposing cultural information to the public can only be identified and assessed by people who are familiar with it and can contextualise its social effect. The only way to be certain that the publication of native title records containing cultural information will not have undue negative consequences is to seek advice directly from the Aboriginal or Torres Strait Islander people concerned. Consultation not only guards against potential hurt or pain, but it also puts control over cultural evidence back in the hands of the people to whom it belongs, reinforcing respectful relationships between Indigenous peoples and government and delivering on the basic intent of the Native Title Act.

\section{Inaccurate, Incomplete or Out-of-Date Information}

The final significant issue associated with creating public access to the NNTT research reports is that they can present a partial or inaccurate impression of the native title rights of a particular group if they contain out-of-date, incorrect or misleading information. The misunderstandings such information may perpetuate can have a significant impact on relationships and reputations, and can generate argument and conflict among and between individuals and groups.

The NNTT research reports were prepared for a very specific tasknamely, to provide information to assist with the mediation and resolution of native title claims. They were designed to be used and considered alongside other forms of evidence-namely, the testimony of contemporary claimants. They were not written with a general public readership in mind, and as noted above are limited in scope and variable in quality. The inclusion of ethno-historical sources in the NNTT research reports is no endorsement of their reliability, and the most accurate and persuasive historical records for a native title claim will not necessarily have been included. Rather than a fulsome account, each research report

29 Griffiths $v$ Northern Territory of Australia (No 3) [2016] 900 FCA 318. 
offers only a glimpse of the reality of Aboriginal and Torres Strait Islander connections to country as understood through only a fraction of the total body of relevant evidence. ${ }^{30}$ Additionally, as soon as there is any change to the status of the underlying claim, the research reports are effectively out-of-date and are unlikely to reflect the facts of native title as the Federal Court has subsequently determined them to be.

All of this makes for a knowledge asset that, despite its inherent value, is nevertheless highly problematic and open to misinterpretation. The possibility for NNTT research reports and other native title records to contribute to social conflict is often raised during public forums and private discussions about the management of native title information. ${ }^{31}$ As an Aboriginal colleague who has been closely involved in her own family's native title claim explained:

I have seen traditional owners have different views on research materials and in most cases have seen conflicts created due to the lack of proper understanding of the research material [in circumstances where] traditional owners and community doubt the reliability of the primary and secondary sources.

All that [the research reports] provide is a snapshot analogy of cultural and historical bibliographic research that was done on their traditional country and I believe it gives a very marginal understanding of the authenticity of the research. ${ }^{32}$

30 This broader body of evidence would usually include oral testimony of claimants; genealogies; contemporary maps of boundaries and sites of significance; and difficult-to-obtain but more in-depth ethno-historical materials, such as anthropological field notes held in international archives. The Federal Court and NNTT do not hold copies of all the evidence that may have been submitted in relation to native title applications resolved by consent determination. However, state governments will hold copies of this material. Given the large number of native title claims to date that have been resolved through mediation rather than litigation, the collections of native title records held by state governments will be much larger than those held by the Federal Court and NNTT. They too, presumably, are struggling with the challenge of how to manage them.

31 For example, see proceedings of a workshop on native title information management hosted by AIATSIS in 2015; McGrath et al, above n 4.

32 Email to author, 10 February 2016. The source wishes to remain anonymous. 
It is difficult to assess the likelihood of public access to the NNTT research reports or other native title records creating or aggravating social conflict. ${ }^{33}$ In each instance, the possibility will be context-dependent and will be influenced to a great extent by the circumstances of those concerned at any given moment in time. Even if the likelihood of social conflict is low, the potential severity of its consequences warrants careful consideration as to whether the educational benefits that potentially flow from creating public access ultimately outweigh the associated risks.

That the contents of research reports may be unreliable, incomplete or misleading raises a compelling argument that on that basis alone they should not be made publicly available. That their publication may also contribute to social conflict and damage to the reputations of Aboriginal and Torres Strait Islander peoples and their relationships with others tips the scales even further in that direction.

Nevertheless, it should not be presumed that native title groups will always want to prevent the publication of NNTT research reports. The same colleague who raised concerns about their potential to generate arguments also expressed a belief that they should be made publicly available in some form so that traditional owners can use them to help locate, review and understand historical information relevant to their people and country. ${ }^{34}$ The issue is, then, one of consent.

\section{Informed Consent and the Need for Meaningful Consultation}

On the face of it, the risks associated with creating public access to the NNTT research reports are likely much reduced because most of the information they contain is already in the public domain. However, this assumption fails to consider the circumstances in which that information was initially collected, and, in particular, whether free, prior informed consent for recording and distribution was provided by the original informants.

33 For further discussion of the generative role of native title research in social change and conflict, see Simon Correy, 'The Reconstitution of Aboriginal Sociality through the Identification of Traditional Owners in New South Wales' (2006) 17(3) The Australian Journal of Anthropology 336; Eve Vincent, Against Native Title: Conflict and Creativity in Outback Australia (Aboriginal Studies Press, 2017).

34 Correy, above n 33; Vincent, above n 33. 
Since the introduction of the Native Title Act in 1993, many thousands of Aboriginal and Torres Strait Islander people have willingly shared information of a personal or culturally sensitive nature with third parties to progress their native title claims. However, the NNTT research reports also include cultural and personal information that was not collected with the consent of named individuals or groups. While this may not be an issue when using this information for a native title claim, it becomes one when it is to be made available for public use.

Determining the existence of prior consent requires examination of the expectations of the informants at the time that the information was originally collected. Establishing consent in circumstances where everyone involved in the original exchange-researchers and informants alike-may have long since passed away is especially difficult. Even when information has been more recently gathered and from a consenting individual for the express purpose of providing evidence for a native title claim, agreement to use that information for a secondary purposenamely public education—cannot be assumed.

The impossibility of establishing consent does not negate the need for consultation with relevant persons or groups. Rather, circumstances where consent is either absent or ambiguous call for more, not less, consultation about the risks of making sensitive information publicly available. For although it may seem that any damage would already have been done as a result of someone else publishing the material, the repetition of that injustice may still impact directly on Aboriginal and Torres Strait Islander peoples.

What does a meaningful and effective consultation look like in the context of native title? In 2010, the then Aboriginal and Torres Strait Islander Social Justice Commissioner, Mick Gooda, suggested that at a minimum it requires that:

- consultation processes should be products of consensus

- consultations should be in the nature of negotiations

- consultations need to begin early and should, where necessary, be ongoing

- Aboriginal and Torres Strait Islander peoples must have access to financial, technical and other assistance

- Aboriginal and Torres Strait Islander peoples must not be pressured into making a decision 
- adequate time frames should be built into consultation processes

- consultation processes should be coordinated across government departments

- consultation processes need to reach the affected communities

- consultation processes need to respect Aboriginal and Torres Strait Islander representative and decision-making structures

- governments must provide all relevant information and do so in an accessible way. ${ }^{35}$

Designing and implementing processes that pay heed to the above criteria and are agreed to by all parties will be neither straightforward nor quick. Key informants may have passed away or be difficult to locate, and other native title groups may hold divergent views about publishing ethnohistorical sources relevant to both. Similarly, descendants of a particular named individual may hold different opinions about how best to manage personal information about a shared ancestor. Like native title claims themselves, the process of figuring out the fate of the many thousands of records they have produced is likely to be a long and expensive one.

\section{Discoverability: A Possible Way Forward}

In the context of limited resources available for consultation and risk mitigation, how should the NNTT and the Federal Court proceed with the challenge of managing the research reports into the future?

One course of action would be to simply deposit them, along with all other native title records, with the NAA under the strictest possible access conditions. The disadvantage of this approach is that it limits the potential educational and historical value of native title records by making it very difficult for members of the public-Indigenous and non-Indigenous alike-to locate and access them. Of greatest concern is that traditional owners and prescribed bodies corporate who do not hold their own copies of these materials may have difficulty accessing them, as will other Aboriginal and Torres Strait Islander persons who, for whatever reason, are not actively involved in their relevant native title group.

35 Australian Human Rights Commission, Native Title Report 2010 (Australian Human Rights Commission, 2011) 60 . 
One way to preserve the broader educative and historical value of native title records while minimising the risks associated with their exposure is to make them publicly discoverable, but not publicly accessible. By 'discoverable', I mean that information about what records exist and the conditions under which they may be accessed can be easily and readily found by anyone who is looking for them. This approach would ensure that until such time that meaningful consultation with individuals and groups can occur, circulation of the records themselves is limited but knowledge of their existence is not lost.

To this end, the NNTT has recently investigated the feasibility of creating an online map-based search tool that would enable users to generate bibliographies of native title research assets, including the research reports and the ethno-historical items they contain. Copies of the assets themselves would not be provided unless the consent of the native title group, identifiable persons and copyright holders has been obtained.

Online technologies and reliable standardised metadata will be crucial to the success of this strategy. Much of the contextual information currently contained in the research reports-namely, background information about the identity and qualifications of authors and the circumstances in which they collected their data-could readily be included in the metadata for each record, without risk of exposing personal or culturally sensitive information. This would assist the reader with assessing the reliability and relevance of particular documents without having to view the originals.

The ambition to create discoverability does, nevertheless, raise a critical question about the extent to which the act of collating and publishing metadata about native title records is in and of itself an act that requires consultation with Aboriginal and Torres Strait Islander peoples. The risks are certainly much reduced; however, any consequences will still be experienced almost exclusively by Indigenous Australians alone, suggesting some form of consultation-perhaps with prescribed bodies corporate and/or native title representative bodies-may be warranted before proceeding with this strategy. 


\section{Conclusion}

All of the issues discussed above in relation to the management of NNTT research reports are relevant to the development of management policies for other types of native title records held by the NNTT and the Federal Court—for example, court transcripts, genealogies, preservation evidence and connection reports. Because these documents were created exclusively for native title proceedings and contain original historical, cultural and personal information unavailable elsewhere, these particular records are potentially of far greater value than the research reports. The potential benefits of making them publicly available will be greater, but then so too will the associated risks, and these risks will be disproportionately experienced by Aboriginal and Torres Strait Islander peoples.

In the development of policies to identify and mitigate these risks, the possible impacts of any particular course of action on the relationships and levels of trust between Indigenous Australians and government should not be forgotten. For when these relationships fail, it is invariably Aboriginal and Torres Strait Islander people who suffer. The recent report Overcoming Indigenous Disadvantge: Key Indicators 2016 noted that 'stronger relationships between Aboriginal and Torres Strait Islander Australians and non-Indigenous Australians build and sustain mutual respect, while mutual respect contributes to stronger relationships-[it is] a virtuous circle' ${ }^{36}$ The report further noted that participation in decision-making was a key element of self-determination, which, in turn, is a critical part of governance. Effective governance, leadership and recognition of culture are essential to the social and economic development of Aboriginal and Torres Strait Islander Australians and have the potential to influence virtually all other indicators of wellbeing. ${ }^{37}$

If we recognise that a fundamental intention of the Native Title Act is to provide for the advancement and protection of Aboriginal and Torres Strait Islander peoples, and to progress the process of reconciliation among all Australians, then it is incumbent on those of us who are responsible for developing policy to put those interests and relationships at the centre of all decisions about the fate of native title records.

36 Productivity Commission, Overcoming Indigenous Disadvantage: Key Indicators 2016 (Commonwealth of Australia, 2016) 7 <http://www.pc.gov.au/research/ongoing/overcoming-indigenous -disadvantage/2016\#thereport>.

37 Ibid 25-6. 
A robust approach to developing policy to assess and mitigate risks associated with creating public access to native title records is one that prioritises the interests of Aboriginal and Torres Strait Islander peoples. It is one that recognises that what is legally possible is not necessarily the most appropriate way forward, and, instead, invests in consultation towards achieving informed consent. However, where the resources are not available to facilitate this level of engagement, providing for public discoverability of native title records is a viable alternative that will ensure the inherent value of these precious knowledge assets is preserved until issues of public access can be resolved. 
This text is taken from The Court as Archive, edited by Ann Genovese, Trish Luker and Kim Rubenstein, published 2019 by ANU Press, The Australian National University, Canberra, Australia. doi.org/10.22459/CA.2019.09 